\title{
WEAK AND POINTWISE COMPACTNESS IN THE SPACE OF BOUNDED CONTINUOUS FUNCTIONS
}

BY

ROBERT F. WHEELER ${ }^{1}$

\begin{abstract}
Let $T$ be a completely regular Hausdorff space, $C_{b}(T)$ the space of bounded continuous real-valued functions on $T, M(T)$ the Banach space dual of $C_{b}(T)$. Let $\mathcal{H}$ denote the family of subsets of $C_{b}(T)$ which are uniformly bounded and relatively compact for the topology $\sigma_{p}$ of pointwise convergence. The basic question considered here is: what is the largest subspace $Z$ of $M(T)$ such that every member of $\mathcal{H}$ is relatively $\sigma\left(C_{b}, Z\right)$-compact? Classical results of Grothendieck and Ptak show that $Z=M(T)$ if $T$ is pseudocompact. In general, $M_{t} \subset Z \subset M_{s}$; assuming Martin's Axiom, a deep result of Talagrand improves the lower bound to $\boldsymbol{M}_{\tau}$. It is frequently, but not always, true that $\boldsymbol{Z}=\boldsymbol{M}_{\boldsymbol{s}}$; counterexamples are given which use Banach spaces in their weak topologies to construct the underlying $T$ 's.
\end{abstract}

A well-known theorem of Grothendieck [28] asserts that if $T$ is a compact Hausdorff space, and $H$ is a uniformly bounded, pointwise compact set of real-valued continuous functions on $T$, then $H$ is compact in the weak topology of the Banach space $C(T)$. Thus, in this case, the weak and pointwise topologies agree on $H$.

This article is concerned with extensions of Grothendieck's Theorem to the setting: $T$ is a completely regular Hausdorff space, and $C_{b}(T)$ is the Banach space of bounded continuous real-valued functions on $T$, with the supremum norm. Ptak [51] and Tomasek [64] have shown that Grothendieck's Theorem as stated above holds if and only if $T$ is pseudocompact. However, one can pursue the matter as follows:

Main Problem. For a given completely regular $T$, what is the largest linear subspace $Z$ of $M(T)$, the Banach space dual of $C_{b}(T)$, such that the pointwise topology and the weak topology $\sigma\left(C_{b}, Z\right)$ agree on uniformly bounded, pointwise compact sets of continuous functions?

Now $Z$ is easily seen to be a norm-closed band in the Banach lattice $M(T)$. The thrust of this work is to position $Z$ with respect to the subspaces of $M(T)$ commonly encountered in topological measure theory: the spaces $M_{t} \subset M_{\tau} \subset M_{s}$ $\subset M_{\sigma}$ of tight, $\tau$-additive, separable, and $\sigma$-additive Baire measures, respectively. It is an easy consequence of Grothendieck's Theorem that $M_{t} \subset Z$ for all $T$. But one can do better: a deep result of Talagrand [62], which assumes Martin's Axiom, leads to a proof that $M_{\tau} \subset Z$.

Received by the editors July 29, 1980.

1980 Mathematics Subject Classification. Primary 46A50, 28C15; Secondary 46E10, 54D60.

${ }^{1}$ Partially supported by NSF Grant MCS-8002523. 
On the other hand, considerations involving equicontinuous subsets of $C_{b}(T)$ show that $Z \subset M_{s}$. Indeed in a great many cases, $Z=M_{s}$. Much of our effort is devoted to determining when this strongest possible result holds. It is true, for example, if $T$ is metrizable, or if $C_{b}(T)$ is angelic [50] in the pointwise topology. Haydon [30] and Pryce [50] give very general conditions under which $C_{b}(T)$ has the angelic property.

However, it is not always true that $Z=M_{s}$. In particular, this fails if $T$ is the closed unit ball of the Banach space $C\left[0, \omega_{1}\right]$, with the relative weak topology. The pointwise compact $H$ involved here is the dual unit ball. A similar result holds for the closed unit ball of $l^{\infty}=C(\beta N)$, with the topology of pointwise convergence on $\beta N$. Here an example of Fremlin and Talagrand [23] yields a separable measure which is not even countably continuous (in the sense of Ptak [51]) on a pointwise compact and separable $H$. The analysis suggests a close relationship between the space $Z$ and the Pettis integrability of bounded weakly measurable functions from a probability space into a Banach space.

Pointwise compactness in the larger space $C(T)$ of all continuous real-valued functions on $T$ has been studied extensively (see, for example, [15], [30], [41], [50], [65], [68]). We draw some connections between results of these authors and those given here, and resolve a conjecture of Haydon and Kirk: pointwise compactness does not always "go up" from $T$ to its Dieudonné completion. The paper concludes with a list of open questions.

1. Basic definitions and terminology. Throughout, $T$ denotes a completely regular Hausdorff space. $\mathcal{T}_{p}$ (or $\mathcal{T}_{p}(T)$, if confusion might arise) is the topology on $C_{b}(T)$ of pointwise convergence on $T$. Let $\mathcal{E}$ (resp., $\mathcal{H}$ ) denote the family of uniformly bounded subsets of $C_{b}(T)$ which are equicontinuous (resp., relatively $\mathcal{T}_{p}$-compact). Then $\mathcal{E} \subset \mathcal{H}$, and equality holds if and only if $T$ is discrete (see [40, Theorem 1.6]). A subset $A$ of $C_{b}(T)$ is relatively $\mathscr{T}_{p}$-countably compact if every sequence in $A$ has a $\mathcal{T}_{p}$-cluster point in $C_{b}(T)$.

A good reference for the duality theory of locally convex spaces is Schaefer [55]. If $\langle E, F\rangle$ is a dual pair of vector spaces, then $\sigma(E, F)$ and $\tau(E, F)$ denote the weak and Mackey topologies on $E$ with respect to $F$.

A basic reference for topology is the Gillman-Jerison text [25]. We require some facts about the structure of $\beta T$, the Stone-Čech compactification of $T$. A subset $B$ of $T$ is said to be bounded in $T$ (or relatively pseudocompact) if the restriction to $B$ of any $f \in C(T)$ is bounded. Then $\mu T$ is the smallest subspace of $\beta T$ which contains $T$ and has the property that its bounded subsets have compact closure. Buchwalter [11] gives a construction of $\mu T$; see also [8], [10], [30], [34]. $T$ is said to be a $\mu$-space if $T=\mu T$. The Nachbin-Shirota Theorem asserts that $C(T)$, with the compact-open topology, is a barrelled LCS if and only if $T$ is a $\mu$-space. Tweddle [65, Theorem 10] has a related result. All $P$-spaces [25], metacompact normal spaces, and Banach spaces with their weak topologies [66] are $\mu$-spaces.

Also important is $\theta T$, the topological space underlying the completion of $T$ in the finest compatible uniform structure. It is referred to variously as the Dieudonné completion, universal completion, or $c$-repletion of $T$. Every paracompact space is 
Dieudonné complete. See [10], [14] for additional details. The Hewitt realcompactification $\nu T$ of $T$ is treated extensively in [25], [69].

In general, $T \subset \mu T \subset \theta T \subset \nu T \subset \beta T$. The equality $\mu T=\beta T(\theta T=\beta T, \nu T=$ $\beta T$ ) holds if and only if $T$ is pseudocompact (i.e., every continuous real-valued function on $T$ is bounded). Barring the existence of 2 -valued measurable cardinals, $\theta T=\nu T$ for every $T[25$, Chapter 15$]$.

If $f \in C(T)$, then $f^{\nu}$ is the unique continuous extension to $\nu T$. If $H \subset C(T)$, then $H^{\nu}=\left\{f^{\nu}: f \in H\right\}$. Also, $\{t: f(t)=0\}$ is called a zero set of $T$; its complement is called a cozero set. The Baire (Borel) sets of $T$ are the least $\sigma$-algebra of subsets of $T$ containing the zero sets (closed sets). If $A \subset T$, then $\operatorname{cl}_{\beta T} A$ is the closure of $A$ in $\beta T$.

Let $M(T)$ denote the dual space of $\left(C_{b}(T),\|\|_{\infty}\right)$. We adopt the Alexandroff representation for $M(T)$ as the space of nonnegative, finite, finitely-additive, zero-set regular Baire measures on $T$ [67]. If $p \in \beta T$, then $\delta(p)$, the point mass at $p$, is the member of $M(T)$ defined by $\delta(p)(Z)=1$, if $Z$ is a zero set of $T$ such that $p \in \mathrm{cl}_{\beta T} Z$, and 0 otherwise.

We distinguish the linear subspaces $L(T) \subset M_{t}(T) \subset M_{\tau}(T) \subset M_{s}(T) \subset M_{\sigma}(T)$ of $M(T)$. Here $L(T)$ is the linear span of $\{\delta(p): p \in T\}$, so that $\mathcal{T}_{p}=\sigma\left(C_{b}, L\right)$. For a discussion of the tight, $\tau$-additive, and $\sigma$-additive Baire measures, see [41], [43], [67]. The separable measures $M_{s}(T)$ were introduced by Dudley [17] and are studied in [31], [32], [41], [59], [74]. If $\mu \in M(T)$, then $\mu$ is a separable measure if and only if the restriction of $\mu$ to any member of $\mathcal{E}$ is $\mathcal{T}_{p}$-continuous.

Many relationships among these classes of measures are known. For example, $M_{t}=M_{\tau}$ if and only if $T$ is measurable with respect to every regular Borel measure on $\beta T$ (e.g., if $T$ is completely metrizable or locally compact). $M_{\tau}=M_{s}$ if $T$ is paracompact [74], in particular if $T$ is metrizable. Barring the existence of real-valued measurable cardinals, $M_{s}=M_{\sigma}$ for every $T$ (see [59, Theorem 3.1]). Also, $M_{s}$ (or $M_{\sigma}$ ) is equal to $M$ if and only if $T$ is pseudocompact.

The $\{0,1\}$-valued members of $M, M_{\sigma}, M_{s}$, and $M_{\tau}$ correspond to points of $\beta T, \nu T, \theta T$, and $T$, respectively (see 7.3 for a question regarding $\mu T$ ). Thus if $M_{\sigma}=M_{\tau}$ (resp., $M_{s}=M_{\tau}$ ), then $T$ is realcompact (resp., Dieudonné complete), but not conversely [47].

Topoplogies $\beta_{0}, \beta$, and $\beta_{1}$ on $C_{b}(T)$ which yield $M_{t}, M_{\tau}$, and $M_{\sigma}$, respectively, as dual spaces are introduced in [24], [58]. It is shown in [74] that $\beta_{e}=\tau\left(C_{b}, M_{s}\right)$ is the finest locally convex topology on $C_{b}(T)$ which agrees with $\mathscr{T}_{p}$ on every member of $\varepsilon$.

\section{Measures which are continuous on $\mathcal{T}_{p}$-compact sets.}

DEFINITION 2.1. $Z=\{\mu \in M(T)$ : the restriction of $\mu$ to any member of $\mathcal{H}$ is $\mathcal{T}_{p}$-continuous $\}$.

Clearly $L(T) \subset Z$. It is straightforward to verify that $Z$ is the largest linear subspace $Y$ of $M(T)$ such that $\mathcal{T}_{p}$ and $\sigma\left(C_{b}, Y\right)$ agree on members of $\mathcal{H}$. Moreover, $Z$ is the largest linear subspace $W$ of $M(T)$ such that $W \supset L(T)$, and each member of $\mathcal{H}$ is relatively $\sigma\left(C_{b}, W\right)$-compact. 
In this section we record some preliminary results on $Z$, and give a short proof of the Ptak-Tomasek result mentioned in the introduction. Note that $M(T)$ is a Banach lattice under the total variation norm.

THEOREM 2.2. $Z$ is a norm-closed linear subspace of $M(T)$, and a band [55], hence is generated by its positive cone.

Proof. It is immediate that $Z$ is a norm-closed linear subspace of $M(T)$. Now let $\mu \in Z, H \in \mathcal{K}$, and $f \in C_{b}(T)$. If $\left(h_{\alpha}\right)$ is $\mathcal{T}_{p}$-convergent to $h_{0}$ in $H$, then $f \cdot\left(H-h_{0}\right)$ is in $\mathcal{K}$, and so $\mu_{f}\left(h_{\alpha}-h_{0}\right)=\mu\left(f h_{\alpha}-f h_{0}\right) \rightarrow 0$. Thus $\mu_{f} \in Z$. By [59, Theorem 1.2], $Z$ is a band in $M(T)$.

It is well known that $M_{t}, M_{\tau}, M_{s}$, and $M_{\sigma}$ have the properties listed in 2.2. $M_{\sigma}$ and $M_{s}$ have the additional property of being weak*-sequentially complete [67, Theorem 19], [74, Proposition 4.4]. Whether this is true for $Z$ is not known to the author (see 7.2).

The next result is known (see, for example, [26], [35], [41]), but we include a proof for completeness.

THEOREM 2.3. $M_{t} \subset Z \subset M_{s}$ for every space $T$.

Proof. If $\mu \in M_{t}(T)$, then $\mu$ has a unique extension to a compact-regular Borel measure on $T$. Let $H \in \mathcal{H}$ and $f_{\alpha} \rightarrow f_{0}$ in $\left(H, \mathcal{T}_{p}\right)$. Given $\varepsilon>0$, choose a compact subset $K$ of $T$ such that $|\mu|(T-K)<\varepsilon / 4 M$, where $M=\sup \left\{\|f\|_{\infty}: f \in H\right\}$. Restricting $H$ to $K$, Grothendieck's Theorem [28, Théorème 5] for compact spaces yields an $\alpha_{0}$ such that $\left|\int_{K}\left(f_{\alpha}-f_{0}\right) d \mu\right|<\varepsilon / 2$ for $\alpha \geqslant \alpha_{0}$. It follows that $\left|\int_{T}\left(f_{\alpha}-f_{0}\right) d \mu\right|<\varepsilon$ for $\alpha \geqslant \alpha_{0}$. Hence $\mu$ is $\mathcal{T}_{p}$-continuous on $H$, so $\mu \in Z$.

Since $\mathcal{E} \subset \mathcal{H}$, each $\mu \in Z$ is $\mathcal{T}_{p}$-continuous on uniformly bounded equicontinuous sets, hence is a member of $M_{s}(T)$.

Thus, for a given $T$, the best possible answer to the Main Problem is that $Z=M_{s}$. This is true whenever $T$ is metrizable, as we now show.

THEOREM 2.4. If $T$ is metrizable, then every uniformly bounded, relatively $\mathfrak{T}_{\boldsymbol{p}}$ countably compact subset $H$ of $C_{b}(T)$ is relatively $\sigma\left(C_{b}, M_{s}\right)$-compact.

Proof. Let $\left(f_{n}\right)$ be a sequence in $H$, and let $\mu_{1}, \ldots, \mu_{n} \in M_{\tau}(T)$. There is a closed separable subset $S$ of $T$ such that $\left|\mu_{i}\right|(T-S)=0 \forall i$ [67]. Let $\left(s_{i}\right)$ be dense in $S$, and let $g$ be a $\mathcal{T}_{p}$-cluster point of $\left(f_{n}\right)$ in $C_{b}(T)$. By the diagonal procedure, there is a subsequence $\left(f_{n}^{\prime}\right)$ such that $\lim _{n} f_{n}^{\prime}\left(s_{i}\right)=g\left(s_{i}\right)$ for all $i$. Now if $h \in C_{b}(T)$ is any $\sigma_{p}$-cluster point of $\left(f_{n}^{\prime}\right)$, then $h$ and $g$ agree on all $s_{i}$, hence on $S$. It follows that $f_{n}^{\prime} \rightarrow g$ pointwise on $S$.

Now let $\varepsilon>0$. By the Lebesgue Convergence Theorem, there is an $n_{0}$ such that $\left|\mu_{i}\left(f_{n}^{\prime}\right)-\mu_{i}(g)\right|<\varepsilon$ for all $n \geqslant n_{0}$ and all $i$. Thus $g$ is a $\sigma\left(C_{b}, M_{\tau}\right)$-cluster point of $\left(f_{n}\right)$, so $H$ is relatively $\sigma\left(C_{b}, M_{\tau}\right)$-countably compact. Since $\left(C_{b}(T), \beta\right)$ is a complete LCS for metrizable $T$ [24, Theorem 7], $H$ is relatively $\sigma\left(C_{b}, M_{\tau}\right)$-compact. But $M_{\tau}=M_{s}$ for metrizable $T$, and the result follows.

A space $T$ is metacompact if every open cover has an open point-finite refinement. The best source of information for measures on metacompact spaces is [48]. 
Corollary 2.5. If $T$ is metacompact and locally metrizable, then $M_{\tau} \subset Z$.

Proof. Let $H \in \mathcal{H}$, and let $\mu \in M_{\tau}(T)$. Then $\mu$ has a closed-regular Borel extension [43]. The support $S$ of $\mu$ is Lindelöf [48] and locally metrizable, hence metrizable by a well-known result of Smirnov. Now $H_{1}=\{h \mid S: h \in H\}$ is uniformly bounded and relatively $\mathcal{T}_{p}(S)$-compact, and $\mu \mid S$ is $\tau$-additive. An application of 2.4 completes the proof.

A metacompact locally metrizable space need not be metrizable (see [63]). Assuming Martin's Axiom, 2.5 is a special case of Theorem 3.2 below.

TheOREM 2.6 [PTAK-TOMASEK]. $Z=M(T)$ if and only if $T$ is pseudocompact.

Proof. If $Z=M(T)$, then $M_{s}(T)=M(T)$, by 2.3. Thus $\theta T=\beta T$, and so $T$ is pseudocompact. Conversely, suppose $T$ is pseudocompact, and let $H \in \mathcal{H}$. For each sequence $\left(f_{n}\right)$ in $H$ and $p \in \nu T$, there is a point $t \in T$ such that $f_{n}^{\nu}(p)=f_{n}(t)$ for all $n[25,7 \mathrm{C}]$. If follows that $H^{\nu}$ is $\mathcal{T}_{p}(\nu T)$-countably compact. But $\nu T=\beta T$ is compact, and the elegant proof of Grothendieck's Theorem due to Rosenthal [53, 3.2] now shows that $H$ is $\sigma\left(C_{b}, M\right)$-sequentially compact. By Eberlein's Theorem, $H$ is $\sigma\left(C_{b}, M\right)$-compact, so $Z=M(T)$.

3. The separation property and angelic spaces. Much outstanding work on pointwise compact sets of continuous (or measurable) functions has been published recently, although not in the context of our Main Problem. In this section we draw on these results to sharpen our information about $Z$.

Let $(\Omega, \Sigma, \mu)$ be a finite measure space, and let $H$ be a $\mathscr{T}_{p}(\Omega)$-compact set of real-valued measurable functions on $\Omega$. Then $H$ has the "separation property" [36] with respect to $\mu$ if whenever $h_{1}, h_{2} \in H$ and $h_{1}=h_{2}$ a.e. $(\mu)$, then $h_{1}(\omega)=h_{2}(\omega)$ for all $\omega \in \Omega$. Let $\mathcal{T}_{\mu}$ be the topology on $H$ of convergence in $\mu$-measure. $\left(H, \mathcal{T}_{\mu}\right)$ is Hausdorff if and only if $H$ has the separation property; in this case, $\sigma_{\mu}>\sigma_{p}$ on $H$, and $\left(H, \mathscr{T}_{\mu}\right)$ is completely metrizable [62].

Alexandra Bellow [36]-[38] has demonstrated the usefulness of the "separation property", and has asked for conditions on $(\Omega, \Sigma, \mu)$ and $H$ which will insure, in its presence, that $\mathcal{T}_{p}=\mathcal{T}_{\mu}$ on $H$. We summarize some of the best results to date on this problem.

Proposition 3.1. Assume that $H$ is $\mathcal{T}_{p}$-compact and has the separation property with respect to $\mu$. Then $\mathcal{T}_{p}=\mathcal{T}_{\mu}$ on $H$ if any of the following properties hold:

(a) $H$ is $\mathcal{T}_{p}$-sequentially compact (Bellow [37]).

(b) $H$ is convex (Bellow [38]; see also Khurana [39]).

(c) $(\Omega, \Sigma, \mu)$ is a perfect measure space (Fremlin [22]).

(d) always, assuming Martin's Axiom (Talagrand [62]).

Theorem 3.2. Assuming Martin's Axiom, $M_{\tau}(T) \subset Z$ for every $T$.

Proof. Let $\mu$ be a nonzero $\tau$-additive measure on $T$. Then $\mu$ has a nonempty support $S$. If $H \in \mathcal{H}$, then $H_{1}=\{f \mid S: f \in H\}$ is $\mathscr{T}_{p}(S)$-relatively compact. Moreover, $H_{1}$ has the separation property with respect to $\mu_{1}=\mu \mid S$, because all members of $H$ are continuous, and spt $\mu_{1}=S$. From Talagrand's result 3.1(d), 
$\left(H_{1}, \mathcal{T}_{p}(S)\right)$ is a compact metric space, and it follows that $\mu_{1}$ is $\mathscr{T}_{p}(S)$-continuous on $H_{1}$. Hence $\mu$ is $\mathscr{T}_{p}(T)$-continuous on $H$, and so $M_{\tau} \subset Z$.

Even without Martin's Axiom, it can frequently be shown that $M_{\tau} \subset Z$. This is certainly true if $M_{t}=M_{\tau}$ (for example, if $T$ is locally compact), using $2.3 ; 2.5$ is another positive re ult. A space $T$ is a $k_{R}$-space if the continuity of a real-valued function on $T$ is guaranteed by its continuity on compact subsets. It is known [58] that $\left(C_{b}(T), \beta_{0}\right)$ is a complete LCS if (and only if) $T$ is a $k_{R}$-space. Now if $H \in \mathcal{H}$, then $H$ is relatively $\sigma\left(C_{b}, M_{t}\right)$-compact, by 2.3 . Hence if $T$ is $k_{R}$, then the convex hull of $H$ is again relatively $\sigma\left(C_{b}, M_{t}\right)$-compact, and Bellow's result 3.1(b), combined with the argument of 3.2, shows that $M_{\tau} \subset Z$. (See 6.2 for another application of 3.1(b).)

Let $M_{p}(T)$ denote the space of perfect Baire measures on $T$ [22], [23], [44], [54]. Every 2-valued measure is perfect, and $M_{t} \subset M_{p} \subset M_{\sigma}$, but neither $M_{p}$ nor $M_{\tau}$ contains the other in general. Fremlin's result 3.1(c), combined with the argument of 3.2, shows that $M_{p} \cap M_{\tau} \subset Z$. However, Example 5.4 shows that $M_{p} \not Z$ in general, i.e., a $\sigma\left(C_{b}, M_{t}\right)$-compact set need not be $\sigma\left(C_{b}, M_{p}\right)$-compact. Also $Z \varnothing$ $M_{p}$ : consider a $\tau$-additive, nontight measure on a separable metric space.

Suppose now that $H \in \mathcal{H}$ and $\mu \in M_{s}(T)-M_{\tau}(T)$; when will $\mu$ be $\mathcal{T}_{p}$-continuous on $H$ ? The argument of 3.2 will not work, because the support of $\mu$ may well be empty. This happens, for example, if $\mu=\delta(p)$, where $p \in \theta T-T$. For a less trivial example, let $T$ be the Sorgenfrey plane. This space is Dieudonné complete, yet Moran [47] constructs on it a nonzero separable measure with empty support.

One could try to restore the separation property as follows: given $\mu \in M_{s}(T)$ and $H \in \mathcal{K}$, define $h_{1} \sim h_{2}$ if $h_{1}=h_{2}$ a.e. ( $\mu$ ). If $H_{1} \subset H$ can be chosen in such a way that $H_{1}$ is $\mathcal{T}_{p}$-compact and contains exactly one member of each equivalence class, then $H_{1}$ will have the $\mu$-separation property, and the argument of 3.2 will work.

Unfortunately this procedure fails in the simplest nontrivial case. S. Graf has kindly communicated to the author the following example: let $T=\{0,1\}$, so that $C(T)=R^{2}$. Let $H=\left(\left[0, \frac{1}{2}\right] \times\{0\}\right) \cup\left(\left[\frac{1}{2}, 1\right] \times\{1\}\right)$, and let $\mu=\delta(0) \in M_{t}(T)$. Then $H$ is $\mathcal{T}_{p}$-compact, and $(x, y) \sim\left(x^{\prime}, y^{\prime}\right)$ if and only if $x=x^{\prime}$. It is easy to see that any $H_{1} \subset H$ which contains exactly one member of each equivalence class cannot be $\mathcal{T}_{p}$-compact.

In spite of these remarks, there is a large class of spaces $T$ for which $Z=M_{s}$ ( $=M_{\sigma}$ ), the best possible result. A Hausdorff space $S$ is angelic [50] if the closure of each relatively countably compact subset $A$ of $S$ is compact, and consists precisely of the limits of sequences from $A$.

THEOREM 3.3. If $C_{b}(T)$ is $\sigma_{p}$-angelic, then $Z=M_{s}(T)=M_{\sigma}(T)$.

Proof. If $H$ is uniformly bounded and $\mathscr{T}_{p}$-compact, then $H$ is $\mathscr{T}_{p}$-sequentially compact (by the angelic property), and so is $\sigma\left(C_{b}, M_{\sigma}\right)$-sequentially compact by the Lebesgue Convergence Theorem. But the angelic property is preserved by passage to finer regular topologies, and so $H$ is $\sigma\left(C_{b}, M_{\sigma}\right)$-compact. The result follows. 
Applying results of Haydon [30] and Pryce [50], and the fact that any subspace of an angelic space is angelic, we have

COROLlaRY 3.4. If $T$ has a dense $\sigma$-bounded subset, then $C_{b}(T)$ is $\mathcal{T}_{p}$-angelic, and $Z=M_{s}=M_{\sigma}$.

This holds, in particular, if $T$ has a dense pseudocompact or $\sigma$-compact subset (e.g. for $T$ separable).

$T$ is said to have the discrete countable chain condition (DCCC) if every discrete family of open sets is countable (discrete = each point of $T$ has a neighborhood meeting at most one member of the family). It is equivalent to require that each continuous metric image of $T$ be separable [72]. All Lindelöf spaces have the DCCC.

THEOREM 3.5. Each of the following conditions implies the next:

(a) $C_{b}(T)$ is $\mathcal{T}_{p}$-angelic.

(b) $T$ has the DCCC.

(c) $M_{s}(T)=M_{\sigma}(T)$.

Proof. (a) $\Rightarrow$ (b) If not, there is a continuous pseudometric $d$ on $T$ such that $T$ is not $d$-separable. Choose a subset $\left(t_{\alpha}\right)_{\alpha<\omega_{1}}$ of $T$ and $\delta>0$ such that $d\left(t_{\alpha}, t_{\beta}\right) \geqslant \delta$ for $\alpha \neq \beta$ (here $\omega_{1}$ is the first uncountable ordinal). Let $f_{\alpha}(t)=\sup \left(\delta / 3-d\left(t, t_{\alpha}\right), 0\right)$, and let $H=\left\{g_{\beta}\right\}_{\beta<\omega_{1}}$, where $g_{\beta}=\Sigma_{\alpha<\beta} f_{\alpha}$. Then $H \subset C_{b}(T)$ is $\mathcal{T}_{p}$-relatively sequentially compact (and equicontinuous), and $g_{\omega_{1}}$ is in the $\sigma_{p}$-closure of $H$, but is not the $\mathscr{T}_{p}$-limit of any sequence in $H$. Hence $C_{b}(T)$ is not $\mathscr{T}_{p}$-angelic.

(b) $\Rightarrow$ (c) Any space with the DCCC is a $D$-space in the sense of Granirer [27]. Now apply, for example, Theorem 3.1 of [59].

Neither of these implications can be reversed; moreover, the conditions $Z=M_{s}$ and DCCC are unrelated. Indeed a discrete space of cardinal $\kappa_{1}$ satisfies $M_{t}=Z$ $=M_{s}=M_{\sigma}$, but fails the DCCC. Example 5.4 has even the countable chain condition for open sets (using the proof of 5.3), but fails $Z=M_{s}$. Example 4.4 has the DCCC and satisfies $Z=M_{s}=M_{o}$, but $C_{b}(T)$ is not $\mathcal{T}_{p}$-angelic.

4. Some examples. In this section we analyze five of the standard counterexamples of topological measure theory, bringing out various aspects of the Main Problem. The key examples involving Banach spaces with their weak topologies are deferred to $\$ 5$.

EXAMPLE 4.1. $T=\left[0, \omega_{1}\right]$, the (compact) space of ordinal numbers less than or equal to the first uncountable ordinal. Let $H=\left\{\chi_{\{\alpha\}}: \alpha<\omega_{1}\right.$, $\alpha$ a nonlimit ordinal $\} \cup\{0\}$. It is easy to see that $H \in \mathcal{H}$. If $d$ is a continuous pseudometric on $T$, then there is an $\alpha_{0}<\omega_{1}$ such that $d(\alpha, \beta)=0$ for all $\alpha, \beta>\alpha_{0}$. Thus $H$ is not a subset of the $d$-continuous (or even $d$-Borel measurable) functions on $T$. Hence $\mathcal{T}_{p}$-compact sets do not match up with continuous pseudometrics in the same nice way that equicontinuous sets do (see [74, Proposition 3.1]). Kirk [40, Theorem 2.4] has a positive result on "control" of pointwise compact sets of continuous functions. 
EXAMPLE 4.2. $T=[0,1]$, with the right half-open interval topology [60, Example 51]. $T$ is Lindelöf, hence realcompact. The Baire and Borel sets in $T$ coincide with the Euclidean Borel sets. Lebesgue measure on $T$ is not tight, since compact subsets of $T$ are countable. We have

$$
l^{1}(T)=M_{t} \varsubsetneqq M_{\tau}=Z=M_{s}=M_{\sigma}=M_{t}[0,1] .
$$

As a corollary, a uniformly bounded, $\mathcal{T}_{p}$-compact set of right-continuous functions on $[0,1]$ is compact in the weak topology determined by $M_{t}[0,1]$. This is a special case of more general results (concerning Baire class 1 functions on Polish spaces) due to Rosenthal [52] and others.

EXAMPLE 4.3. $T$ is a space originally introduced by Negrepontis, used in [70] to give the first example of a locally compact nonparacompact space such that $\left(C_{b}(T), \beta\right)$ is a Mackey space. The continuum hypothesis is assumed in the construction of $T$. As noted in [70], $T$ is a $\mu$-space, but not Dieudonné complete $(\theta T-T$ is a single point $p)$. Since $T$ is separable, $C_{b}(T)$ is $\sigma_{p}$-angelic, by 3.4 , and so $M_{t}=M_{\tau} \varsubsetneqq Z=M_{s}=M_{\sigma}$. The codimension of $M_{\tau}$ in $Z$ is 1 .

Since $C_{b}(T)$ is $\sigma\left(C_{b}, M_{s}\right)$-angelic, every relatively countably compact subset of $C_{b}(T)$ has compact closure in this topology. This is true in spite of the fact that $\beta_{e}=\tau\left(C_{b}, M_{s}\right)$ is not quasi-complete [70]. The point $p \in \theta T-\mu T$, yet $\delta(p)$ is $\mathcal{T}_{p}$-continuous on members of $\mathcal{H}$. The completion of $\left(L, \tau\left(L, C_{b}\right)\right)$ is $\left(M_{s}, \tau\left(M_{s}, C_{b}\right)\right)$. This shows that $M_{\tau}=M_{t}$ need not be complete in the Mackey topology $\tau\left(M_{t}, C_{b}\right)$, even for locally compact $T$. ( $\$ 6$ contains additional discussion of this point.)

Example 4.4. $T$ is the non-Dieudonne complete $P$-space given in $[25,9 \mathrm{~L}] . T$ is a $\mu$-space, but not Dieudonné complete $\left(\theta T-T=\left\{\omega_{2}\right\}\right)$. Haydon [30] has shown that $\mathcal{T}_{p}(T)$-compact subsets of $C(T)$ are $\mathcal{T}_{p}(\theta T)$-compact. Using general results for $P$-spaces [73], it follows that

$$
l^{1}(T)=M_{t}(T)=M_{\tau}(T) \varsubsetneqq Z=M_{s}(T)=M_{\sigma}(T)=l^{1}(\theta T) .
$$

As in 4.3, the codimension of $M_{\tau}$ in $Z$ is 1 .

Now $\theta T$ is Lindelöf; since any space and its Dieudonné completion have the same continuous metric images [14], $T$ has the DCCC. Yet $C_{b}(T)$ is not $\mathcal{T}_{p}$-angelic: $H_{0}=\left\{f \in C_{b}(T):\|f\|_{\infty} \leqslant 1\right\}$ is $\mathcal{T}_{p}$-closed and countably compact, but not compact [73, Theorem 2.3]. The point $\omega_{2} \in \theta T-\mu T ; \delta\left(\omega_{2}\right)$ is $\mathcal{T}_{p}$-continuous on members of $\mathcal{H}$, but not on $H_{0}$.

Additional information about $T$ appears in $\$ 4$ of [73]. See 7.5 of this paper for a remark about Dieudonné complete $P$-spaces.

Example 4.5. $T$ is the Dieudonné plank [60, Example 89], [71], [75]. Then $T$ is metacompact, but not Dieudonné complete: $\theta T=T \cup\{p\}$, where $p=\left(\omega_{1}, \omega_{0}\right)$ is the corner point. The $\{0,1\}$-valued separable Baire measure $\delta(p)$ is very poorly behaved in at least two senses: it admits no countably-additive extension to the Borel sets of $T[71,4.1]$, and has no countably-additive preimage on $E(T)$, the projective cover of $T$ [75, Example 1]. However, it does belong to $Z$, and consequently $Z=M_{s}$ for this space. To see this, suppose there is a member $H$ of 
$\mathcal{H}, f_{0} \in H, G \subset H$, and $\varepsilon>0$ so that $f_{0}$ is in the $\mathcal{T}_{p}$-closure of $G$ and $\mid f^{\theta}(p)-$ $f_{0}^{\theta}(p) \mid \geqslant \varepsilon$ for all $f \in G$. Since the maps $f \rightarrow f-f_{0}, f \rightarrow a \wedge(f \vee b), f \rightarrow c f$ are $\mathcal{T}_{p}$-continuous for any $a, b, c \in R$, we may assume that $f_{0} \equiv 0$ and $f^{\theta}(p)=1$ for all $f \in G$. For each $n$, choose $f_{n} \in G$ such that $\left|f_{n}\left(\omega_{1}, i\right)\right|<1 / n, 1<i \leqslant n$. There is an $\alpha_{0}<\omega_{1}$ such that (1) $\left|f_{n}(\alpha, i)\right|<1 / n$ for $\alpha_{0} \leqslant \alpha \leqslant \omega_{1}, 1 \leqslant i \leqslant n$, and all $n$; and (2) $f_{n}\left(\alpha, \omega_{0}\right)>1-1 / n$ for $\alpha_{0} \leqslant \alpha<\omega_{1}$ and all $n$. Now $\left(f_{n}\right)$ has a $\mathcal{T}_{p}$-cluster point $g_{0}$ in $H \subset C_{b}(T)$. But $g_{0}\left(\alpha_{0}, \omega_{0}\right)=1$, while $g_{0}\left(\alpha_{0}, n\right)=0$ for all $n$. This contradicts the continuity of $g_{0}$ at $\left(\alpha_{0}, \omega_{0}\right)$.

5. Banach spaces, and counterexamples to the conjecture $Z=M_{s}$. Throughout this section, $X$ (or $(X$, weak)) denotes a Banach space with its weak topology $\sigma\left(X, X^{*}\right)$. Let $B_{X}=\{f \in X:\|f\| \leqslant 1\}$. Similarly, $B_{X}$. and $B_{X} *$ are the closed unit balls of the dual and bidual, respectively.

Corson [13] studied the topological properties of ( $X$, weak). In particular, he showed that $\nu X$ can be realized as a linear subspace of $X^{* *}$, with the relative weak*-topology. Valdivia [66] has shown that $X=\mu X$ always holds. Additional details about ( $X$, weak) can be found in recent work of Edgar [18], [19]. In particular, $X$ can always be embedded as a dense subspace of $R^{A}$ for suitable $A$ (e.g., a Hamel basis for $X^{*}$ ), and so $X$ has the countable chain condition (CCC) on open sets. The Baire sets of $X$ coincide with the $\sigma$-algebra generated by the members of $X^{*}[18,2.3]$.

Lemma 5.1. $M_{t}(X)=M_{\tau}(X) \subset Z \subset M_{s}(X)=M_{o}(X)$ for every $X$.

Proof. Tortrat has shown that $M_{t}=M_{\tau}$ (see $\left.[18,3.5]\right)$. Since $X$ has the CCC, and therefore the DCCC, $M_{s}=M_{\sigma}$ must hold by 3.5 .

Thus $\theta X=\nu X$ for all $X . X \subsetneq \theta X$ can occur (Example 5.4). Edgar [18, 5.4] has characterized the condition $M_{\sigma}(X)=M_{\tau}(X)$ in terms of vector-valued measurable functions into $X$. We shall have more to say about this in $\$ 7$.

At this point we offer a short, purely topological proof of Corson's characterization of $\nu X$, using methods due to Blair and Hager. We refer to [6], [7] for the notions of $z$-embedding, $O z$-space, and $G_{\delta}$-closure.

Theorem 5.2 (Corson). $\nu X=\left\{\phi \in X^{* *}\right.$ : for each countable subset $C$ of $X^{*}$, $\exists f \in X$ such that $\phi|C=f| C\}$, with the relative $\sigma\left(X^{* *}, X^{*}\right)$ topology.

Proof. Since ( $X^{* *}$, weak ${ }^{*}$ ) is a dense subspace of $R^{A}$ for suitable $A, 5.3(\mathrm{a})$ and 5.6 of [6] show that it is an $O z$-space. Thus from 5.1(d) and 5.3(a) of the same paper, $\left(X\right.$, weak) is an $O z$-space; also, it is $z$-embedded in $\left(X^{* *}\right.$, weak $)$, which is $\sigma$-compact, hence realcompact. By $[7,2.6] \nu X$ is the $G_{\delta}$-closure of $X$ in $\left(X^{* *}\right.$, weak*). It is easy to see that the $G_{\delta}$-closure of $X$ is exactly the space described in the hypothesis.

A compact Hausdorff space $K$ is scattered if it has no nonempty perfect subsets. Such a space admits no nonatomic regular Borel measures (see [57]). Thus if $X=\left(C(K),\|\|_{\infty}\right)$, then $X^{*}=M(K)=l^{1}(K)$ and $X^{* *}=l^{\infty}(K)$. 
TheOREM 5.3. Let $X=C(K)$, where $K$ is compact and scattered, and $X$ has the weak topology. Then:

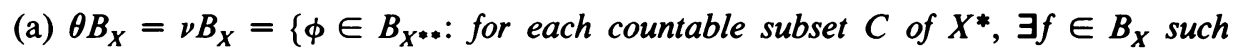
that $\phi|C=f| C\}$, with the relative $\sigma\left(X^{* *}, X^{*}\right)$ topology.

(b) $B_{X}$ is $C$-embedded in $X[25]$.

(c) $X$ is realcompact if and only if $B_{X}$ is realcompact.

Proof. (a) $B_{X^{* *}}=\left\{\phi \in l^{\infty}(K):|\phi(k)| \leqslant 1 \forall k \in K\right\}=[-1,1]^{K}$ is an $O z$-space $[6,5.6]$. Thus $B_{X}$ is $z$-embedded in $B_{X^{* *}}$, and the characterization of $\nu B_{X}$ follows as in 5.2. (b) The map $f \rightarrow-1 \vee(f \wedge 1)$ is a continuous retraction of $X$ onto $B_{X}$, each endowed with the topology of pointwise convergence on $K$. But for scattered $K$, the weak topology coincides with the pointwise topology on $B_{X}$; hence the map is a retraction for the respective weak topologies. Clearly, then, any continuous realvalued function on $\left(B_{X}\right.$, weak) admits a continuous extension to $(X$, weak), so we have (b). Moreover, existence of the retraction shows that $\left(B_{X}\right.$, weak) has the CCC, since ( $X$, weak) does. Thus $\theta B_{X}=\nu B_{X}$, to complete the proof of (a). (c) Half of this is clear, since a closed subset of a realcompact space is realcompact [25]. Conversely, (b) implies that each multiple of $B_{X}$ is $z$-embedded in $X$, and $[6,3.9]$ completes the proof.

Note that a space which is a countable or even finite union of closed realcompact subspaces need not be realcompact, $([6,3.10]$, or Example 4.5 of this paper).

Now we are ready for the promised example of a space $T$ which fails $Z=M_{s}$. Note that in the construction, measures become functions, and functions become measures.

EXAMPLE 5.4. Let $X=C\left[0, \omega_{1}\right]$, with the weak topology, and let $T=B_{X}$. Let $H=B_{X^{*}}$, with the relative weak*topology. Then $H$ is a uniformly bounded, $\sigma_{p}$-compact absolutely convex subset of $C_{b}(T)$.

Now $X$ is not realcompact [18]. Since $\left[0, \omega_{1}\right]$ is scattered, $B_{X}$ is also not realcompact (5.3(c))). By 53(a), the function $\phi$ in $X^{* *}=l^{\infty}\left[0, \omega_{1}\right]$ given by $\phi(\alpha)=$ $0, \alpha<\omega_{1}, \phi\left(\omega_{1}\right)=1$ is in $\nu T-T=\theta T-T$. Thus $\mu=\delta(\phi)$ is a $\{0,1\}$-valued separable (perfect) measure on $T$. But $\mu$ is not $\mathcal{T}_{p}$-continuous on $H$ : for each $\alpha \leqslant \omega_{1}$, let $f_{\alpha} \in C_{b}(T)$ be the function on $T$ corresponding to the point mass $\delta(\alpha) \in X^{*}=l^{1}\left[0, \omega_{1}\right]$. Then $\left\{f_{\alpha}\right\} \subset H$, and $\lim _{\alpha \rightarrow \omega_{1}} f_{\alpha}=f_{\omega_{1}}$ in $\mathcal{T}_{p}\left(=\sigma\left(X^{*}, X\right)\right)$, but $\mu\left(f_{\alpha}\right)=\langle\phi, \delta(\alpha)\rangle=\phi(\alpha)=0, \alpha<\omega_{1}$, and 1, $\alpha=\omega_{1}$. Hence $Z \subsetneq M_{s}$.

A similar example can be constructed for any scattered $K$ such that $C(K)$ is not weakly realcompact. The final example of the section exhibits a far more subtle way in which the condition $Z=M_{s}$ may fail.

EXAMPLE 5.5. Let $X=l^{\infty}=C(\beta N)$, with the topology of pointwise convergence on $\beta N$, and let $T=B_{X}$. Then $T$ admits a coarser compact metric topology $\left(\sigma\left(l^{\infty}, l^{1}\right)=\right.$ pointwise convergence on $\left.N\right)$, so $T$ is hereditarily realcompact [25, 8.18]. For each $p \in \beta N$, the point mass $\delta(p)$ in $X^{*}$ corresponds to $f_{p} \in C_{b}(T)$, and $H=\left\{f_{p}: p \in \beta N\right\}$ is a uniformly bounded $\mathscr{T}_{p}$-compact separable subset of $C_{b}(T)$.

Fremlin and Talagrand [23, 2B(b)] construct a $\sigma$-algebra $\Sigma$ of subsets of $2^{N}$ and a probability measure $\bar{\mu}$ on $\Sigma$ such that the map $A \rightarrow \chi_{A}: 2^{N}=\mathscr{P}(N) \rightarrow l^{\infty}$ is 
measurable from $\Sigma$ to the Baire $\sigma$-algebra of $\left(l^{\infty}\right.$, weak), but not Pettis integrable. As in the proof of 5.3(b), $T$ is $C$-embedded in $l^{\infty}$, each having the topology $\mathcal{T}$ of pointwise convergence on $\beta N$. Thus for every Baire set $B$ of $(T, \mathcal{T})$, there is a Baire set $C$ of $\left(l^{\infty}, \mathcal{T}\right)$ such that $C \cap T=B$. Since $\mathcal{T}$ is coarser than the weak topology on $l^{\infty}$, it follows that $A \rightarrow \chi_{1}: 2^{N}=\mathscr{P}(N) \rightarrow T$ is measurable from $\Sigma$ to the Baire $\sigma$-algebra of $(T, \mathcal{T})$. Let $\lambda$ be the image measure of $\bar{\mu}$ under $\chi$. Then $\lambda \in M_{\sigma}(T)$, and indeed $\lambda \in M_{s}(T)$ (argue as in the proof of 5.3, using the pointwise topology on $T$ and $l^{\infty}$, to see that $T$ has the CCC; apply Theorem 3.5).

Now the calculations in [23] show that if $n \in N$,

$$
\int_{T} f_{n} d \lambda=\int_{2^{N}} f_{n} \circ \chi d \bar{\mu}=\bar{\mu}\{A: n \in A\}=\frac{1}{2}
$$

while if $p \in \beta N-N$, then

$$
\int_{T} f_{p} d \lambda=\int_{2^{N}} f_{p} \circ \chi d \bar{\mu}=\vec{\mu}(\mathscr{F})=1
$$

where $\mathcal{F}$ is the free ultrafilter on $N$ corresponding to $F$.

Thus $\lambda$ is not $\mathcal{T}_{p}$-continuous on $H$, so $Z \varsubsetneqq M_{s}$. Indeed $\lambda$ is not even $\mathcal{T}_{p}$-countably continuous [51] on $H$, i.e., it fails to preserve cluster points of sequences. For further discussion of this pathology, see $\$ 6$.

6. Pointwise compactness in $C(T)$. Since Grothendieck's original paper [28], pointwise compactness in $C(T)$, the space of all real-valued continuous functions on $T$, has been examined repeatedly. We refer the reader to Ptak [51], DeWilde [15], Haydon [30], [32], Kirk [41], Pryce [50], Schmets [56], Tweddle [65], and Vidossich [68], among others, for details. Constantinescu [12] treats the subject in a more general context. Arhangel'skii [2]-[4] gives a deep analysis of the topological structure of $\left(C(T), \mathcal{T}_{p}\right)$.

If $T \subset S \subset \nu T$, let $M_{c}(S)$ denote the space of bounded regular Borel measures $\lambda$ on $\beta T$ such that the support of $\lambda$ is a compact subset of $S$. Then the positive linear functionals on $C(T)$ correspond to members of $M_{c}^{+}(\nu T)$ [41], so that $M_{c}(\nu T) \subset$ $M_{\sigma}(T)$ is the largest reasonable collection of measures to consider in this context.

If $S$ is any completely regular space, then a function $\phi: S \rightarrow R$ is said to be countably continuous on $S$ [51] if whenever $\left(s_{n}\right)$ clusters to $s_{0}$ in $S$, then $\phi\left(s_{n}\right)$ clusters to $\phi\left(s_{0}\right)$. Any sequentially continuous function is countably continuous, but not conversely.

Proposition 6.1. (a) (PTAK [51, 4.1]). Each member of $M_{c}(\nu T)$ is $\mathcal{T}_{p}$-continuous on $\mathcal{T}_{p}$-compact, separable, absolutely convex subsets of $C(T)$.

(b) (PTAK [51, 4.2]). Each member of $M_{c}(\nu T)$ is $\mathcal{T}_{p}$-countably continuous on $\mathcal{T}_{p}$-compact absolutely convex subsets of $C(T)$.

(c) (HAYDON [32]). $\left\{\lambda \in M_{c}(\nu T): \lambda\right.$ is $\mathcal{T}_{p}$-continuous on each $\mathcal{T}_{p}$-bounded equicontinuous subset of $C(T)\}=M_{c}(\theta T)=M_{s} \cap M_{c}(\nu T)$.

(d) (HAYDON [30, 2.9]). If $H \subset C(T)$ is $\mathcal{T}_{p}(T)$-compact, then $H^{\mu}$ is $\mathcal{T}_{p}(\mu T)$-compact. 
Berezanskii [5], Kirk [41] and Tweddle [65] have employed a dual approach to problems of this type, analyzing the completions of $L(T)$ and $M_{c}(T)$ relative to various locally convex topologies on those spaces. In particular, Kirk shows (Theorem 9.5) that the completion of $L(T)$ for the topology of uniform convergence on $\mathcal{T}_{p}$-bounded equicontinuous subsets of $C(T)$ is $M_{s c}=M_{s} \cap M_{c}(\nu T)$. Using Grothendieck's Completeness Theorem, this is precisely the dual of Haydon's result 6.1(c).

How do the examples in $\$ 5$ relate to these results? Propostion 6.1(a) tells us that in any example such as 5.4, where the measure in question is a point mass, $H$ cannot also be required to be $\sigma_{p}$-separable. As for $6.1(\mathrm{~b})$, absolute convexity is used by Ptak to force uniform boundedness of $\mathcal{T}_{p}$-compact sets of functions on compact subsets of $\nu T$. Thus the expected analogue of $6.1(\mathrm{~b})$ for $C_{b}(T)$ might be: "each member of $M_{\sigma}(T)$ is $\sigma_{p}$-countably continuous on uniformly bounded, $\sigma_{p}$-compact subsets of $C_{b}(T)$ ", but this is just what 5.5 denies.

Haydon has (implicitly) asked if 6.1(d) can be improved to read: if $H \subset C(T)$ is $\mathcal{T}_{p}(T)$-compact, then $H^{\theta}$ is $\mathcal{T}_{p}(\theta T)$-compact. Kirk [41, p. 27] conjectures that the completion of $(L, \tau(L, C))$ is $M_{s c}=M_{c}(\theta T)$. The dual formulation is that $\{\lambda \in$ $M_{c}(\nu T): \lambda$ is $\mathcal{T}_{p}$-continuous on each $\mathcal{T}_{p}$-compact absolutely convex subset of $C(T)$ \} should be precisely $M_{c}(\theta T)$. However, Example 5.4 shows that these conjectures are not valid, since the $H$ in question is absolutely convex, and the measure is a point mass from $\theta T$.

We do have

THEOREM 6.2 The completion of $\left(L, \tau\left(L, C_{b}\right)\right)$ always contains $M_{\tau}$.

Proof. The completion is $\left\{\lambda \in M(T): \lambda\right.$ is $\mathcal{T}_{p}$-continuious on $\mathcal{T}_{p}$-compact absolutely convex subsets of $\left.C_{b}(T)\right\}$. Any such set is uniformly bounded [41, 10.2], and Proposition 3.1(b), combined with the argument of Theorem 3.2, completes the proof.

This is a partial answer to another question raised by Kirk [41, p. 28]. Again, Example 5.4 shows that the completion can be properly smaller than $M_{s}(T)$.

We close the section with a few remarks on sequential compactness. It is clear from the Lebesgue Convergence Theorem that any uniformly bounded $\mathcal{T}_{\boldsymbol{p}}$-sequentially compact subset of $C_{b}(T)$ is $\sigma\left(C_{b}, M_{\sigma}\right)$-sequentially compact. It is known [21] that any compact space of cardinal less than $2^{*_{1}}$ is sequentially compact. In fact, under Martin's Axiom, any compact space of cardinal less than $2^{c}$ is sequentially compact [46]. See [20], [45], [61] for additional information. The upshot is that in looking for a measure $\lambda$ and $H \in \mathcal{H}$ such that $\lambda \mid H$ is not $\mathcal{T}_{p}$-countably continuous, it is reasonable to consider $H$ 's of cardinal at least $2^{c}$, such as homeomorphs of $\beta N$. This is exactly the situation of Example 5.5.

7. Some open problems. 7.1. What is the finest locally convex topology on $C_{b}(T)$ which agress with $\mathcal{T}_{p}$ on members of $\mathcal{H}$ ? Conjecture: It is the topology of uniform convergence on weakly compact subsets of the Banach space $Z$ (i.e., $\tau\left(Z^{*}, Z\right) \mid C_{b}$ ). Grothendieck [29] proved this for compact $T$, and the result continues to hold for pseudocompact $T$, using 2.6. It is true for $P$-spaces, by 7.5 below. See also [1], especially Corollary 3.11 . 
7.2. Is $Z$ always weak*-sequentially closed in $M(T)$ (equivalently, in $M_{s}(T)$ )? Failing this, is the weak*-sequential closure of $M_{\tau}$ always contained in $Z$ ?

7.3. Give an intrinsic characterization of $Z \cap \beta T=\left\{p \in \beta T: \delta(p)\right.$ is $\mathscr{T}_{p}$-continuous on each $H \in \mathcal{K}\}$. Is it sequentially closed in $\beta T$ ? A related question, posed originally by Buchwalter, asks for an intrinsic characterization of the points of $\mu T$. Haydon's result $6.1(\mathrm{~d})$ shows that $\mu T \subset Z \cap \beta T$; Examples 4.3 and 4.4 show that the inclusion may be strict.

7.4 Let $X=C(K)$, where $K$ is compact and not scattered. Is the map $f \rightarrow-1 \vee$ $(f \wedge 1)$, mentioned in the proof of 5.3 , continuous from $\left(X\right.$, weak) to $\left(B_{X}\right.$, weak)? It is, of course, sequentially continuous. It is well known that the lattice operation $f \rightarrow f^{+}$on $C(K)$ is not weakly continuous.

7.5. The frequency with which $Z=M_{s}$ suggests a theorem of the form: every uniformly bounded $\mathcal{T}_{p}$-compact subset of $C_{b}(T)$ is "almost equicontinuous". Is there a way to make this precise, so that the relation between $Z$ and $M_{s}$ will become more transparent? A number of approaches can be found in the literature: for example, quasi-equicontinuity [40], simple equicontinuity [9], equicontinuity with respect to a lifting topology [37], and equicontinuity on a dense subset of $T$ [49]. Haydon [30, 3.5] shows that a lattice-closed, relatively $\mathcal{T}_{p}$-compact subset of $C(T)$ must be equicontinuous.

Let $\beta_{c}$ denote the finest locally convex topology on $C_{b}(T)$ which arees with $\mathscr{T}_{p}$ on members of $\mathcal{H}$. If $T$ is a nondiscrete, Dieudonné complete $P$-space, then $\mathcal{E}$ is a proper subcollection of $\mathcal{H}$, yet $\beta_{c}=\beta_{e}$. Indeed from results in [73, §3], and the fact that weak and norm compactness coincide in $M_{\tau}(T)=l^{1}(T)$, it can be shown that $\beta_{c}=\beta_{e}=\tau\left(Z^{*}, Z\right) \mid C_{b}$.

7.6. Is Theorem 5.3 true for any Banach space $X$ ? If 5.3(b) fails, is it true that every zero set of $\left(B_{X}\right.$, weak) is the intersection of $B_{X}$ with a zero set of $(X$, weak)? This would be enough to deduce 5.3(c), using [6, 3.9].

7.7. Let $(\Omega, \Sigma, \mu)$ be a probability space, $X$ a Banach space, $f: \Omega \rightarrow X$ a bounded weakly measurable function [16], [18], [19]. Edgar [18, Theorem 5.2] shows that $f$ is weakly equivalent to a Bochner measurable function if and only if the image measure $f(\mu)=\lambda$ is a $\tau$-additive (= tight) Baire measure on $(X$, weak). This is one way for $f$ to be Pettis integrable over $\Omega$; how can it happen in general?

Conjecture. $f$ is Pettis integrable over $\Omega$ if and only if the image measure $\lambda$ is in $Z$ (with respect to $T=(X$, weak)).

In support of this conjecture, suppose that $\lambda$ is a $\mathcal{T}_{p}$-continuous function on $B_{X^{*}} \subset C(T)$, and $x^{* *}$ is the Dunford integral of $f$ over $\Omega$. Then if $x_{\alpha}^{*} \rightarrow x^{*}$ in $\left(B_{X^{*}}\right.$, weak*),

$$
x^{* *}\left(x_{\alpha}^{*}\right)=\int_{\Omega} x_{\alpha}^{*} \circ f d \mu=\int_{T} x_{\alpha}^{*} d \lambda \rightarrow \int_{T} x^{*} d \lambda=x^{* *}\left(x^{*}\right),
$$

so that $x^{* *} \in X$, i.e., $f$ is Pettis integrable over $\Omega$.

This is not quite a proof in one direction, since the members of $B_{X^{*}}$ are not bounded functions on $T$. One needs clarification of the relationship between $X$ and $B_{X}$, as expressed in 7.6. A proof in the other direction would say that proper behavior of $f(\mu)$ on $B_{X^{*}}$ forces proper behavior on all $\mathcal{T}_{p}$-compact subsets of $C_{b}(T)$. 
If the conjecture is correct, then the Pettis Integral Property [19] is equivalent to the condition $Z=M_{s}$ on ( $X$, weak).

AdDed IN PROof. G. Edgar has shown that if $K$ is the Cantor set, then the answer to 7.4 is "no". This result extends to any compact nonscattered space. With regard to 7.6, Edgar has shown that 5.3(a) fails for the long James space, and that $B_{X}$ is not even $z$-embedded in $X=l^{1}(\Gamma)$, card $\Gamma>$ c. Whether 5.3(c) holds for all $X$ remains open. D. Fremlin has suggested an additional question: can Theorem 3.2 be proved without special axioms?

\section{REFERENCES}

1. W. Adamski, P. Gänssler and S. Kaiser, On compactness and convergence in spaces of measures, Math. Ann. 220 (1976), 193-210.

2. A. V. Arhangel'skii, On some topological spaces that occur in functional analysis, Russian Math. Surveys 31 (1976), no. 5, 14-30.

3. On spaces of continuous functions in the topology of pointwise convergence, Soviet Math. Dokl. 19 (1978), 605-609.

4. Structure and classification of topological spaces and cardinal invariants, Russian Math. Surveys 33 (1978), no. 6, 33-96.

5. I. A. Berezanskii, Measures on uniform spaces and molecular measures, Trans. Moscow Math. Soc. 19 (1968), 1-40.

6. R. L. Blair, Spaces in which special sets are z-embedded, Canad. J. Math. 28 (1976), 673-690.

7. R. L. Blair and A. W. Hager, Extensions of zero-sets and of real-valued functions, Math. Z. 136 (1974), 41-52.

8. J. Blasco, On $\mu$-spaces and $k_{R}$-spaces, Proc. Amer. Math. Soc. 67 (1977), 179-186.

9. J. Brace, Convergence on filters and simple equicontinuity, Illinois J. Math. 9 (1965), 286-296.

10. H. Buchwalter, Fonctions continues et mesures sur un espace complètement régulier, Lecture Notes in Math., vol. 331, Springer-Verlag, Berlin and New York, 1973, pp. 183-202.

11. , Parties bornées d'un espace topologique complètement régulier, Initiation à l'Analyse (Sem. Choquet, 9e année), 1969/70, no. 14.

12. C. Constantinescu, Smulian-Eberlein spaces, Comment Math. Helv. 48(1973), 254-317.

13. H. Corson, The weak topology of a Banach space, Trans. Amer. Math. Soc. 101(1961), 1-15.

14. H. Curzer and A. W. Hager, On the topological completion, Proc. Amer. Math. Soc. 56 (1976), 365-370.

15. M. DeWilde, Pointwise compactness in spaces of functions and R. C. James' theorem, Math. Ann. 208 (1974), 33-47.

16. J. Diestel and J. Uhl, Vector measures, Math. Surveys, no. 15, Amer. Math. Soc., Providence, R. I., 1977.

17. R. Dudley, Convergence of Baire measures, Studia Math. 27 (1966), 251-268.

18. G. Edgar, Measurability in a Banach space. I, Indiana Univ. Math. J. 26 (1977), 663-677.

19. __ Measurability in a Banach space. II, Indiana Univ. Math. J. 28 (1979), 559-579.

20. V. Fedorcuk, A compact space having the cardinality of the continuum with no convergent sequences, Math. Proc. Cambridge Philos. Soc. 81 (1977), 177-181.

21. S. Franklin, On two questions of Moore and Mrowka, Proc. Amer. Math. Soc. 21 (1969), 597-599.

22. D. Fremlin, Pointwise compact sets of measurable functions, Manuscripta Math. 15 (1975), 219-242.

23. D. Fremlin and M. Talagrand, $A$ decomposition theorem for additive set-functions, with applications to Pettis integrals and ergodic means, Math. Z. 168 (1979), 117-142.

24. D. Fremlin, D. Garling and R. Haydon, Bounded measures on topological spaces, Proc. London Math. Soc. (3) 25 (1972), 115-136.

25. L. Gillman and M. Jerison, Rings of continuous functions, Springer-Verlag, New York, 1976.

26. I. Glicksberg, Weak compactness and separate continuity, Pacific J. Math. 11 (1961), 205-214.

27. E. Granirer, On Baire measures on D-topological spaces, Fund. Math. 60 (1967), 1-22.

28. A. Grothendieck, Critères de compacité dans les espaces fonctionnels generaux, Amer. J. Math. 74 (1952), 168-186.

29. __ Sur les applications linéaires faiblement compactes d'espaces du type $C(K)$, Canad. J. Math. 5 (1953), 129-173. 
30. R. Haydon, Compactness in $C_{s}(T)$ and applications, Publ. Dép. Math. (Lyon) 9 (1972), 105-113.

31. On compactness in spaces of measures and measure-compact spaces, Proc. London Math. Soc. (3) 29 (1974), 1-16.

32. __ Sur les espaces $M(T)$ et $M^{\infty}(T)$, C. R. Acad. Sci. Paris Sér. A-B 275 (1972), A989-A991.

33. __ Sur un problème de H. Buchwalter, C. R. Acad. Sci. Paris Sér. A-B 275 (1972), A1077-A1080.

34. A-B 276 (1973), A685-A687.

35. J. Hoffman-Jorgensen, Weak compactness and tightness of subsets of $M(X)$, Math. Scand. 31 (1972), 127-150.

36. A. Ionescu Tulcea, On measurability, pointwise convergence and compactness, Bull. Amer. Math. Soc. 80 (1974), 231-236.

37. On pointwise convergence, compactness, and equicontinuity in the lifting topology. I. Z. Wahrsch. Verw. Gebiete 26 (1973), 197-205.

38. On pointwise convergence, compactness, and equicontinuity. II, Advances in Math. 12 (1974), 171-177.

39. S. S. Khurana, Pointwise compactness and measurability, Pacific J. Math. 83 (1979), 387-391.

40. R. B. Kirk, $A$ note on the Mackey topology for $\left(C_{b}(X)^{*}, C_{b}(X)\right)$, Pacific J. Math. 45 (1973), 543-554.

41. , Complete topologies on spaces of Baire measures, Trans. Amer. Math. Soc. 184 (1973),

$1-29$.

42. __ Topologies on spaces of Baire measures, Bull. Amer. Math. Soc. 79 (1973), 542-545.

43. J. Knowles, Measures on topological spaces, Proc. London Math. Soc. 17 (1967), 139-156.

44. G. Koumoullis, On perfect measures (preprint).

45. N. Levine, On compactness and sequential compactnes, Proc. Amer. Math. Soc. 54 (1976), 401-402.

46. V. Malyhin and B. Sapirovskii, Martin's axiom and properties of topological spaces, Soviet Math. Dokl. 14 (1973), 1746-1751.

47. W. Moran, The additivity of measures on completely regular spaces, J. London Math. Soc. 43 (1968), 633-639.

48. __ Measures on metacompact spaces, Proc. London Math. Soc. (3) 20 (1970), 507-524.

49. I. Namioka, Separate continuity and joint continuity, Pacific J. Math. 51 (1974), 515-531.

50. J. D. Pryce, A device of R. J. Whitley's applied to pointwise compactness in spaces of continuous functions, Proc. London Math. Soc. (3) 23 (1971), 532-546.

51. V. Ptak, Concerning spaces of continuous functions, Czechoslovak Math. J. 5(80) (1955), 412-431.

52. H. P. Rosenthal, Pointwise compact subsets of the first Baire class, Amer. J. Math. 99 (1977), $362-378$.

53. (1974), 83-111.

54. V. Sazonov, On perfect measures, Amer. Math. Soc. Transl. (2) 48 (1965), 229-254.

55. H. Schaefer, Topological vector spaces, Springer-Verlag, Berlin and New York, 1971.

56. J. Schmets, Espaces de fonctions continues, Lectures Notes in Math., vol. 519, Springer-Verlag, Berlin and New York, 1976.

57. Z. Semadeni, Banach spaces of continuous functions, Polish Scientific Publishers, Warsaw, 1971.

58. D. Sentilles, Bounded continuous functions on a completely regular space, Trans. Amer. Math. Soc. 168 (1972), 311-336.

59. D. Sentilles and R. F. Wheeler, Linear functionals and partitions of unity in $C_{b}(X)$, Duke Math. J. 41 (1974), 483-496.

60. L. Steen and J. Seebach, Counterexamples in topology, 2nd ed., Springer-Verlag, New York, 1978.

61. A. Szymanski and M. Turzanski, $\beta N$ and sequential compactness, Colloq. Math. 35 (1976), 205-208.

62. M. Talagrand, Solution d'un problème de A. Ionescu-Tulcea, C. R. Acad. Sci. Paris Sér. A-B 283 (1976), 975-978.

63. F. Tall, On the existence of normal metacompact Moore spaces which are not metrizable, Canad. J. Math. 26 (1974), 1-6.

64. S. Tomasek, On a certain class of $\wedge$-structures. I, II, Czechoslovak Math. J. 20(95) (1970), 1-18; 19-33.

65. I. Tweddle, Some results involving weak compactness in $C(X), C(\nu X)$, and $C(X)^{\prime}$, Proc. Edinburgh Math. Soc. 19 (1975), 221-229. 
66. M. Valdivia, Some new results on weak compactness, J. Funct. Anal. 24 (1977), 1-10.

67. V. Varadarajan, Measures on topological spaces, Amer. Math. Soc. Transl. (2) 48 (1965), 161-228.

68. G. Vidossich, On compactness in function spaces, Proc. Amer. Math. Soc. 33 (1972), 594-598.

69. M. Weir, Hewitt-Nachbin spaces, North-Holland Math. Studies, vol. 17, North-Holland, Amsterdam, 1975.

70. R. F. Wheeler, A locally compact nonparacompact space for which the strict topology is Mackey, Proc. Amer. Math. Soc. 51 (1975), 86-90.

71. __ Extensions of a $\sigma$-additive measure to the projective cover, Lectures Notes in Math., vol. 794, Springer-Verlag, Berlin and New York, 1980, pp. 81-104.

72. __ On separable z-filters, General Topology Appl. 5 (1975), 333-345.

73. __ The strict topology for P-spaces, Proc. Amer. Math. Soc. 41 (1973), 466-472.

74. _ The strict topology, separable measures, and paracompactness, Pacific J. Math. 47 (1973), 287-302.

75. Topological measure theory for completely regular spaces and their projective covers, Pacific J. Math. 82 (1979), 565-584.

Department of Mathematical Sciences, Northern Illinois University, DeKalb, Illinois 60115 